\section{Quantifying biased agonism: understanding the links between affinity and efficacy}

\author{
Sudarshan Rajagopal
}

In the March issue of Nature Reviews Drug Discovery, a Perspective article by Kenakin and Christopoulos (Nature Rev. Drug Discov. 12, 205-216; 2013) ${ }^{1}$ reviewed a number of important developments in our understanding of biased agonism at $\mathrm{G}$ protein-coupled receptors (GPCRs; also known as seven transmembrane receptors) over the past decade. In my opinion, the authors have erred in their presentation of which approaches are most useful for measuring biased signalling at GPCRs. Such models can be broadly considered as pharmacological or allosteric models.

In pharmacological models ${ }^{2-5}$ a ligand is considered to have two properties: affinity (the ability of a ligand to form an agonist-receptor complex) and efficacy (the ability of the agonist-receptor complex to result in a downstream signalling response) (FIG. 1a). The stimulus for initiating signalling is related to ligand efficacy and to the concentration of the agonist-receptor complex, and this relationship differs depending upon the pharmacological model chosen to describe the system.

Allosteric models explicitly account for signalling by the formation of an agonistreceptor-transducer ternary complex ${ }^{6-8}$ (FIG. 1 b). Unlike pharmacological models in which the agonist-receptor complex results in a different stimulus depending on the agonist, the ternary complex results in an equal stimulus for signalling regardless of which ligand is used; the efficacy of a ligand is defined by its ability to form this signallingcompetent ternary complex. The formation

a

b

\section{Allosteric models}

Agonist and receptor

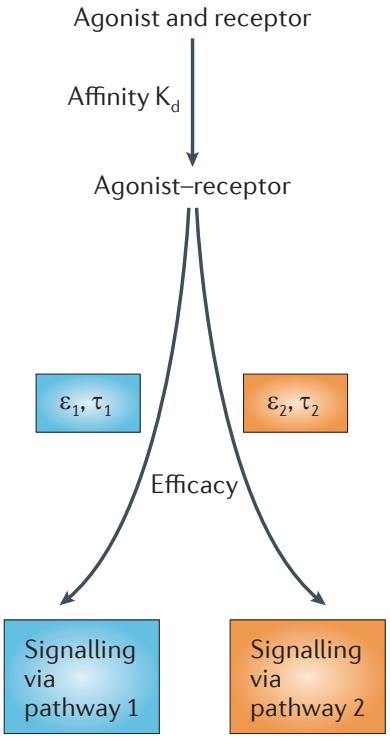

Agonist-receptor-transducer 1
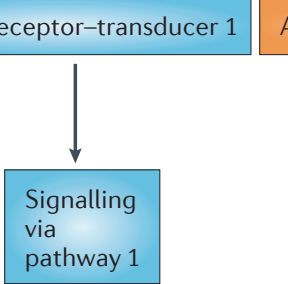

Agonist-receptor-transducer 2

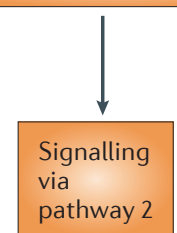

Figure 1 | Pharmacological and allosteric models for interpreting receptor signalling. a | In pharmacological models, formation of an agonist-receptor complex (quantified by affinity) results in signalling, encapsulated by efficacy, such as that described by $\varepsilon$ values or by $\tau$ values. $\mathbf{b} \mid \ln$ an allosteric model, the initial binding of an agonist to the receptor in the absence of transducers occurs in a low-affinity binding state. A high-affinity binding state results from transducer binding and the formation of a ternary complex. In an allosteric model, ligand efficacy can be calculated from the difference between the low-affinity dissociation constant $\left(\mathrm{K}_{\mathrm{d}}\right)$ obtained under conditions that prevent transducer coupling and the affinity associated with transducer $(T)$ coupling, equal to $\mathrm{K}_{\mathrm{d}} /\left(1+\left([T] / \mathrm{K}^{\star}\right)\right)$ in this simplified model. of this ternary complex by an agonist is associated with a high-affinity binding state of the ligand for the receptor-transducer complex that is related to pharmacological efficacy $^{6}$ (FIG. 1).

I believe that a limitation of the approach favoured by Kenakin and Christopoulos to measure biased agonism is that they use a pharmacological model (the operational model) but use an allosteric 'conditional affinity' (that is, an estimate of the affinity of a ligand for a GPCR when a G protein transducer is bound that attempts to account for formation of a ternary complex). Although the use of a conditional affinity value compensates for the fact that pharmacological models underestimate receptor occupancy, I believe that the use of a conditional affinity value will necessarily lead to a gross underestimate of agonist efficacy (FIG. 1).

Kenakin and Christopoulos use the operational model to determine so-called transduction coefficients $\left(\tau / \mathrm{K}_{\mathrm{A}}\right.$; the transducer ratio divided by the conditional affinity). Although transduction coefficients can be useful in comparing the relative signalling of ligands, as well as for determining the rank order of biased signalling for a set of biased agonists ${ }^{9}$, they are problematic when trying to measure the efficacy of agonists that produce maximal effects in a given signalling system (full agonists). Fitting concentrationresponse data for full agonists to transduction coefficients is inherently ambiguous, and may require non-physiological constraints to be used, such as setting $\log \mathrm{K}_{\mathrm{A}}$ values to zero so that a fit to the data can be achieved ${ }^{10}$. Under such conditions, the use of a conditional affinity value may become meaningless. Therefore, I believe that the use of transduction coefficients is an erroneous approach to determine ligand bias.

Also, I do not agree with the statement made by the authors that: "The transduction coefficient scale reduces the measurement of agonism to a single number...”. Instead, I believe that the measurement of agonism is encapsulated by the term $\tau$ (transducer ratio) in the operational model described by Black and Leff 5 . Indeed, Kenakin and Christopoulos demonstrate that there are situations in which the transduction coefficient scale lacks utility as an absolute scale of agonism.

\section{Other models to quantify ligand bias}

I believe that other models for quantifying ligand bias are superior to the approach advocated by Kenakin and Christopoulos. I and my co-workers ${ }^{9}$ favour calculating bias factors using one of two approaches. 
The first approach, which Kenakin and Christopolous also note has merit, is based on intrinsic relative activity values. Relative affinity values are calculated from the maximal effect produced by a ligand ( $\mathrm{E}_{\text {max }}$ value) and the concentration of ligand that produces half-maximal response $\left(\mathrm{EC}_{50}\right.$ value) from concentration-response curves that have slopes equal to unity. For agonists that produce concentration-response curves that have slopes of 1 , intrinsic relative activities are identical to transduction coefficients, but the estimates of bias obtained using this approach are not limited by the problematic fitting routine required for transduction coefficients.

A second approach is based on $\tau$ values that are obtained from fitting the operational model using a ligand dissociation constant that is determined under conditions that prevent formation of a ternary complex. This allows affinity and efficacy to be separated in a pharmacological model. While this approach does require a separate dissociation constant to be determined from binding assays, it does yield an estimate of the efficacy of a ligand, $\sigma_{\text {lig }}$. Systematic differences in experimental conditions between signalling and binding assays can cause discrepancies between potencies of partial agonists determined in signalling and binding assays, thereby limiting the utility of this second approach. In the systems to which I have applied this approach, such differences have been minor. However, if larger differences were noted, I would suggest that the operational model should not be used, as partial agonists would not be predicted to cause a large shift in the functional affinity associated with formation of a ternary complex. In this situation, relative activity values could be used ${ }^{9}$.

\section{Moving forward}

At present there is debate as to the optimal methods to use to quantify ligand bias. To move forward, we need to directly compare the different methodologies to the same simulated and real data to assess which approach yields the best estimate of ligand bias, as well as looking towards developing model-free approaches for understanding and quantifying ligand bias.

Sudarshan Rajagopal is at the Department of Medicine, Duke University Medical Center, Durham, North Carolina 27710, USA e-mail: sudarshan.rajagopal@dm.duke.edu

doi:10.1038/nrd3954-c 1 Published online 17 May 2013
1. Kenakin, T. $\&$ Christopoulos, A. Signalling bias in new drug discovery: detection, quantification and therapeutic impact. Nature Rev. Drug Discov. 12 205-216 (2013).

2. Ariens, E. J. Affinity and intrinsic activity in the theory of competitive inhibition. I. Problems and theory. Arch. Int. Pharmacodyn. Ther. 99, 32-49 (1954).

3. Stephenson, R. P. A modification of receptor theory. Br. J. Pharmacol. Chemother. 11, 379-393 (1956).

4. Furchgott, R. F. in Advances in Drug Research (eds Harper, N. J. \& Simmonds, A. B.) 21-55 (Academic Press, 1966).

5. Black, J. W. \& Leff, P. Operational models of pharmacological agonism. Proc. R. Soc. Lond. B Biol. Sci. 220, 141-162 (1983).

6. De Lean, A., Stadel, J. M. \& Lefkowitz, R. J. A ternary complex model explains the agonist-specific binding properties of the adenylate cyclase-coupled $\beta$-adrenergic receptor. J. Biol. Chem. 255, 7108-7117 (1980).

7. Samama, P., Cotecchia, S., Costa, T. \& Lefkowitz, R. J. A mutation-induced activated state of the beta 2 -adrenergic receptor. Extending the ternary complex model. J. Biol. Chem. 268, 4625-4636 (1993).

8. Weiss, J. M., Morgan, P. H., Lutz, M. W. \& Kenakin, T. P. The cubic ternary complex receptor-occupancy model. III. Resurrecting efficacy. J. Theor. Biol. 181, 381-397 (1996).

9. Rajagopal, S. et al. Quantifying ligand bias at seventransmembrane receptors. Mol. Pharmacol. $\mathbf{8 0}$, 367-377 (2011).

10. Kenakin, T. et al. A simple method for quantifying functional selectivity and agonist bias. ACS Chem Neurosci. 3, 193-203 (2012).

Acknowledgements

The author thanks R. Lefkowitz, T. Costa and O. Onaran for invaluable discussions.

Competing interests statement

The author declares no competing financial interests. 